\title{
THE SCALE-UP PROBLEM FOR ION TRANSPORT IN KAOLINITE CLAYS INCLUDING $P H$-DEPENDENCE
}

\author{
Iury Igreja*, Marcio A. Murad*, Sidarta Lima* and Claude Boutin ${ }^{\dagger}$ \\ * Laboratório Nacional de Computação Científica LNCC/MCT \\ Av. Getúlio Vargas 333, 25651-070 Petrópolis, RJ, Brazil \\ e-mail: iuryhai@lncc.br, murad@lncc.br and sidarta@lncc.br, web page: http://www.lncc.br \\ ${ }^{\dagger}$ Univeristé de Lyon, Lab. Geomateriaux, DGCB URA CNRS 1652, \\ Ecole Nationale des Travaux Publics de l'Etat, 69518 Vaulx-en-Velin Cedex, France \\ e-mail: Claude.Boutin@entpe.fr, web page: http://www.entpe.fr
}

Keywords: Electrokinetics, Clay, Homogenization, Danckwerts' boundary conditions, Finite element

Summary. We propose a new multiscale computational model to simulate electrokinetic remediation processes in clayey soils. The macroscopic governing equations are derived using the homogenization procedure applied to the pore-scale description consisting of micro-pores saturated by an aqueous solution containing four monovalent ionic species $\left(\mathrm{Na}^{+}, \mathrm{H}^{+}, \mathrm{Cl}^{-}, \mathrm{OH}^{-}\right)$and charged solid particles surrounded by thin electrical double layers. The homogenized equations are discretized by the finite element method and applied to numerically simulate an electroosmosis experiment for decontamination of a clay sample by electrokinetics. Boundary conditions of Dankwerts type are postulated at the electrodes which brings additional complexity to the iterative algorithm for solving the discrete model.

\section{INTRODUCTION}

In earlier papers $[1,2]$ some of the authors have developed new upscaling methods to describe electrochemical phenomena in active clays. Within the framework of homogenization-based approaches the complex effective response of the medium has incorporated pore-scale electro-chemo-mechanical couplings with phenomena such as electro-osmosis, electro-migration and osmotic swelling naturally appearing in the macroscopic forms of the convection-diffusion equations, Darcy's law and modified Terzaghis effective stress principle.

Despite the enormous improvement achieved by multiscale models they were developed for electrolyte solutions composed of two fully dissociated monovalent ions $\mathrm{Na}^{+}$and $\mathrm{Cl}^{-}$and constant $p H=7$. Consequently the transport of $H^{+}$and $\mathrm{OH}^{-}$ions along with chemical protonation/deprotonation reactions between the $H^{+}$ions and surface charged groups lying on the clay particle surface leading to $\mathrm{pH}$-dependency were neglected. First attempts at incorporating $\mathrm{pH}$ effects within the framework of homogenization were pursued by the authors in $[3,4]$. Numerical approximations of the resultant multiscale model were carried-out and applied to computationally simulate the one-dimensional electro-osmotic experiment with Dirichlet boundary conditions enforced at the electrodes. In this work we generalize these simulations to incorporate more realistic boundary condition of Danckwerts type at the electrodes [5]. The assessment of the numerical results obtained 


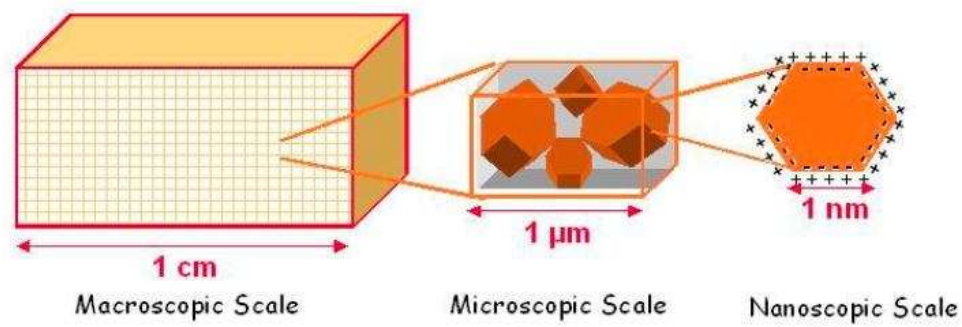

Figure 1: Macroscopic, microscopic and nanoscopic portraits in Kaolinite

within this new computational model is performed through a perturbation-based approach which gives rise to semi-analytical solutions of the electro-osmosis experiment capable of establishing accurate scenarios of electro-kinetic remediation of clayey soils.

\section{REVIEW OF THE MULTISCALE MODEL}

We begin by reviewing the three-scale electro-chemical model (Fig.1) proposed in $[3,4]$ incorporating $p H$-effects. Let $\Omega=\Omega_{s} \cup \Omega_{f} \subset \Re^{3}$ be the microscopic domain occupied by a biphasic porous media composed of clay particles and micro-pores filled by a bulk fluid, and $\Gamma_{f s}$ the solid / fluid interface. The hydrodynamics is governed by the classical Stokes problem

$$
\nabla \cdot \mathbf{v}=0, \quad \mu_{f} \triangle \mathbf{v}-\nabla p=0 \quad \text { in } \Omega_{f}
$$

where $\mathbf{v}$ is the fluid velocity, $p$ the pressure and $\mu_{f}$ the water viscosity. The steady state movement of the four ionic species with concentrations $C_{i}\left(i=\left(\mathrm{Na}^{+}, \mathrm{H}^{+}, \mathrm{Cl}^{-}, \mathrm{OH}^{-}\right)\right.$is governed by the Nernst-Planck equations

$$
\nabla \cdot\left(C_{i b} \mathbf{v}\right)-\nabla \cdot\left[D_{i}\left(\nabla C_{i b}+C_{i b} \nabla \bar{\phi}\right)\right]+\dot{m}=0, \quad\left(\dot{m}=0 \text { for } i=N a^{+}, C l^{-}\right) \quad \text { in } \Omega_{f}
$$

where $D_{i}$ are the binary water-ion diffusion coefficients, $\bar{\phi}:=F \phi / R T$ the dimensionless microscopic electrical potential with $\{F, R, T\}$ the set composed of Faraday constant, universal ideal gas constant and absolute temperature and $\dot{m}$ a source term that quantifies the mass production of $\mathrm{H}^{+}$and $\mathrm{OH}^{-}$due to water hydrolysis.

To close our microscopic model it remains to postulate boundary conditions on the particle/micropore interface $\Gamma_{f s}$. Consider that the normal component of velocity and electro-diffusive ion fluxes vanish on $\Gamma_{f s}$. In addition, owing to the presence of the thin electrical double layer surrounding the particles we postulate the so-called Smoluchowsky slip [2] wherein the tangential velocity exhibits a discontinuity at the interface. Denoting and $\tau$ the unitary tangential vector at $\Gamma_{f s}$ we have

$$
\mathbf{v} \cdot \boldsymbol{\tau}=\widetilde{\epsilon}_{0} \widetilde{\epsilon}_{r} \mu_{f}^{-1} \zeta \nabla \phi \cdot \boldsymbol{\tau} \quad \text { on } \Gamma_{f s}
$$

where $\widetilde{\epsilon}_{0}$ is the permittivity of the free space, $\widetilde{\epsilon}_{r}$ is the dielectric constant of the solvent and $\zeta$ the zeta potential. To close the microscopic model we need to build-up the constitutive law of the $\zeta$-potential 
in terms of $\mathrm{pH}$ and salinity. To this end we make we proceed within the framework of the electrical double layer theory with a nonlinear surface charge density due to protonation / deprotonation chemical reactions. This yields a single algebraic equation for the $\zeta$-potential whcih has been solved in $[3,4]$

The numerical constitutive response $\zeta=\zeta\left(C_{N_{a b}+}, C_{H^{+}}\right)$is depicted in (Fig.2). We may note strong dependence of the $\zeta$-potential on the $p H$ in a nearly skew-symmetric fashion around the point of zero charge (isoelectric point) $p H=5.5$.

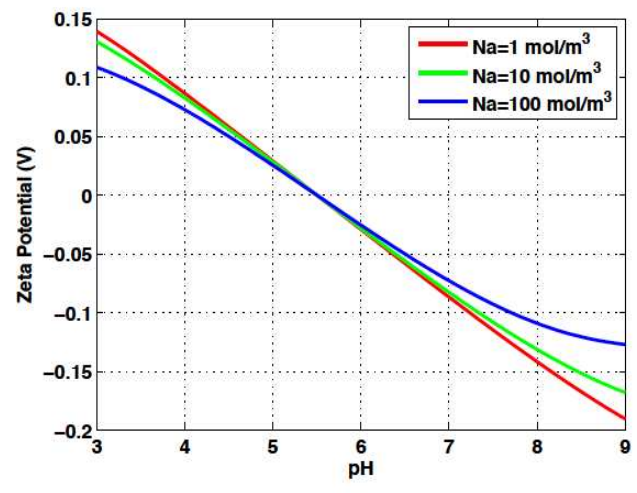

(a)

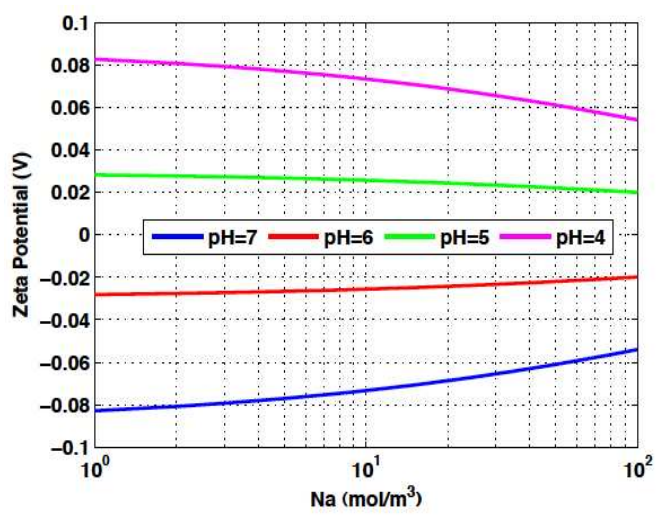

(b)

Figure 2: Constitutive Response of the $\zeta$-Potential as a Function of $p H$ and $C_{\mathrm{Nab}^{+}}$.

Application of the a formal homogenization procedure to the pore-scale model leads to the macroscopic equations [3]

$$
\left\{\begin{array}{l}
\nabla \cdot \mathbf{V}_{\mathbf{D}}=\nabla \cdot\left[-K_{P} \nabla p-K_{E} \nabla \phi\right]=0 \\
\nabla \cdot \mathbf{J}_{\mathbf{N a}^{+}}=\nabla \cdot\left[C_{N a^{+}} \mathbf{V}_{\mathbf{D}}-D_{N a^{+}}\left(\nabla C_{N a^{+}}+C_{N a^{+}} \nabla \bar{\phi}\right)\right]=0 \\
\nabla \cdot \mathbf{J}_{\mathbf{H}^{+}}=\nabla \cdot\left[\Theta C_{H^{+}} \mathbf{V}_{\mathbf{D}}-\widehat{D}_{H^{+}}\left(\nabla_{x} C_{H^{+}}+C_{H^{+}} \nabla \bar{\phi}\right)\right]=0 \\
\nabla \cdot \mathbf{I}_{\mathbf{f}}=\nabla \cdot\left[A \nabla C_{N a b^{+}}+B \nabla C_{H b^{+}}+C \nabla \bar{\phi}\right]=0
\end{array}\right.
$$

where $\mathbf{V}_{\mathbf{D}}$ the Darcy velocity, $\left(K_{P}, K_{E}\right)$ the hydraulic and electroosmotic permeability, $\left(\mathbf{J}_{\mathbf{N a}^{+}}, \mathbf{J}_{\mathbf{H}^{+}}\right)$ the convective/electro-diffusive ionic fluxes, $\left(\Theta, \widehat{D}_{H^{+}}\right)$nonlinear coefficients given by $\Theta:=1-$ $K_{W} / C_{H b^{+}}^{2}$ and $\widehat{D}_{H^{+}}:=D_{H^{+}}+D_{\mathrm{OH}^{-}} K_{W} / C_{H b^{+}}^{2}$, $\mathbf{I}_{\mathbf{f}}$ the electric current, $C$ the electrical conductivity and $(A, B)$ the coupling coefficients for the current $[3,4]$. The electroomsotic permeability is nothing but the average of the $\zeta$-potential and theerfore inherits its constitutive behavior.

\section{APPLICATION TO AN ELECTROOSMOSIS EXPERIMENT}

The macroscopic system is discretized by finite elements and applied to simulate an electroosmosis experiment. An anode and a cathode are placed at the positions $x=0$ and $x=L$ respectively. Denoting $\mathrm{C}_{\mathrm{Na}^{+}}^{r e f}, \mathrm{C}_{\mathrm{H}^{+}}^{r e f}$ the sodium and $\mathrm{H}^{+}$concentration in the reservoir in the anode compartment and $I_{0}$ a given electric current postulate Danckwerts' boundary conditions at the elctrodes [5] 


$$
\begin{array}{ll}
\left.\mathbf{J}_{\mathbf{N a}^{+}}\right|_{x=0}=C_{N a^{+}}^{r e f} \mathbf{V}_{\mathbf{D}} & \left.\mathbf{J}_{\mathbf{N a}^{+}}\right|_{x=L}=C_{N a^{+}} \mathbf{V}_{\mathbf{D}} \\
\left.\mathbf{J}_{\mathbf{H}^{+}}\right|_{x=0}=\Theta C_{H^{+}}^{r e f} \mathbf{V}_{\mathbf{D}}+I_{0} F^{-1} & \left.\mathbf{J}_{\mathbf{H}^{+}}\right|_{x=L}=\Theta C_{H^{+}} \mathbf{V}_{\mathbf{D}}+I_{0} F^{-1}
\end{array}
$$

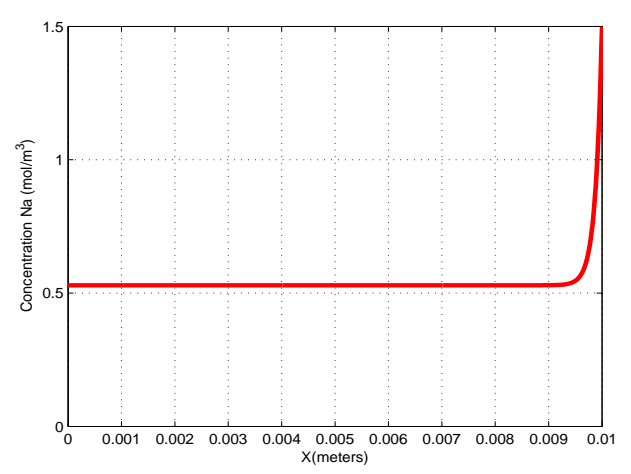

(a)

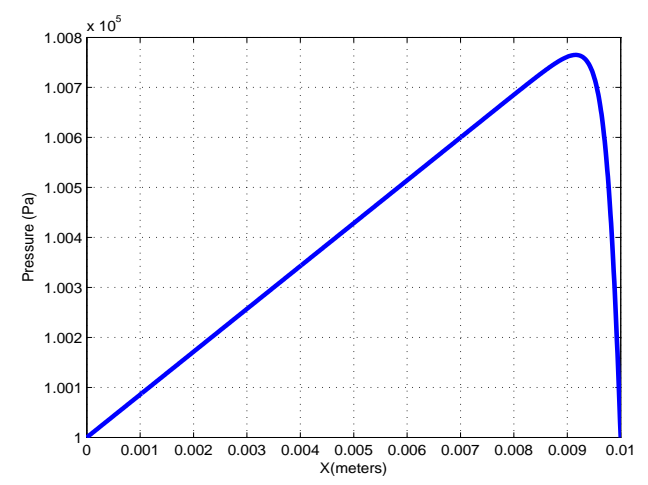

(b)

Figure 3: Sodium and pore pressure steady state profiles

In Fig. 3 we display the sodium concentration and pressure profiles. In contrast to the case of Dirichlet boundary condition presented in [3], the enforcement of (5) leads to a plateau $C_{\mathrm{Na}^{+}}^{\text {platea }}$ (whose value $\mathbf{J}_{\mathrm{Na}^{+}} / \mathbf{V}_{\mathbf{D}}$ can be estimated from perturbation analysis) coupled with a sharp layer in the vicinity of the cathode. In addition, the pressure gradient changes sign to fulfill the incrompressibility constraint. The more realistic simulation presented herein can be further explored to construct reliable electrokinetic remediation scenarios.

\section{REFERENCES}

[1] Moyne, C. and Murad, M., A Two-Scale Model for Coupled Electro-Chemo-Mechanical Phenomena and Onsager's Reciprocity relations in Expansive Clays, Transport in Porous Media, vol. 62(3), pp. 333-380, and vol. 63(1), pp. 13-56 (2006)

[2] Murad, M. A. and Moyne, C., A dual-porosity model for ionic solute transport in expansive clays, Computational Geosciences, vol. 12, pp. 47-82(2008)

[3] Lima, S.A., Murad, M. A., Moyne, C. and Stemmelen, D., A three scale model for pH-dependent steady flows in 1:1 clays, Acta Geotechnica vol. 3, pp. 153-174 (2008)

[4] Lima, S.A., Murad, M. A., Moyne, C. and Stemmelen, D. and Boutin C., A Three-Scale Model of $p H$-dependent flows and Ion Transport with Equilibrium Adsorption in Kaolinite Clays I and II. Transport in Porous Media (2010) (Online first)

[5] Alshawabkeh AN, Acar YB, Electrokinetic remediation: theoretical model. J Geotech Eng, 122:186-196 (1996) 